\title{
Low-level light therapy for treating venous leg ulcers
}

DOI:

10.1002/14651858.CD013061

\section{Document Version}

Final published version

Link to publication record in Manchester Research Explorer

\section{Citation for published version (APA):}

Illescas-montes, R., Atkinson, R. A., \& Cullum, N. (2018). Low-level light therapy for treating venous leg ulcers. Cochrane Database of Systematic Reviews. https://doi.org/10.1002/14651858.CD013061

\section{Published in:}

Cochrane Database of Systematic Reviews

\section{Citing this paper}

Please note that where the full-text provided on Manchester Research Explorer is the Author Accepted Manuscript or Proof version this may differ from the final Published version. If citing, it is advised that you check and use the publisher's definitive version.

\section{General rights}

Copyright and moral rights for the publications made accessible in the Research Explorer are retained by the authors and/or other copyright owners and it is a condition of accessing publications that users recognise and abide by the legal requirements associated with these rights.

\section{Takedown policy}

If you believe that this document breaches copyright please refer to the University of Manchester's Takedown Procedures [http://man.ac.uk/04Y6Bo] or contact uml.scholarlycommunications@manchester.ac.uk providing relevant details, so we can investigate your claim.

\section{OPEN ACCESS}


Cochrane Database of Systematic Reviews

\section{Low-level light therapy for treating venous leg ulcers (Protocol)}

Illescas-Montes R, Atkinson RA, Cullum N

Illescas-Montes R, Atkinson RA, Cullum N.

Low-level light therapy for treating venous leg ulcers.

Cochrane Database of Systematic Reviews 2018, Issue 6. Art. No.: CD013061.

DOI: 10.1002/14651858.CD013061.

www.cochranelibrary.com 


\section{TABLE OF CONTENTS}

HEADER . . . . . . . . . . . . . . . . . . . . . . . . . . . . . . . . . . . . . . . . 1

ABSTRACT . . . . . . . . . . . . . . . . . . . . . . . . . . . . . . . . . . . . . . . 1

BACKGROUND . . . . . . . . . . . . . . . . . . . . . . . . . . . . . . . . . . . . . . 1

OBJECTIVES . . . . . . . . . . . . . . . . . . . . . . . . . . . . . . . . . . . . . .

METHODS . . . . . . . . . . . . . . . . . . . . . . . . . . . . . . . . . . . . . . 4

ACKNOWLEDGEMENTS . . . . . . . . . . . . . . . . . . . . . . . . . . . . . . . . . . . . . . . .

REFERENCES . . . . . . . . . . . . . . . . . . . . . . . . . . . . . . . . . . . . . . . . 7

APPENDICES . . . . . . . . . . . . . . . . . . . . . . . . . . . . . . . . . . . . . . . . . . . . . . . .

CONTRIBUTIONS OF AUTHORS . . . . . . . . . . . . . . . . . . . . . . . . . . . . . . . . . . . . . . 11

DECLARATIONS OF INTEREST . . . . . . . . . . . . . . . . . . . . . . . . . . . . . . . . . . . . . . . 11

SOURCES OF SUPPORT . . . . . . . . . . . . . . . . . . . . . . . . . . . . . . . . . . . . . . 11 
[Intervention Protocol]

\title{
Low-level light therapy for treating venous leg ulcers
}

\author{
Rebeca Illescas-Montes ${ }^{1}$, Ross A Atkinson ${ }^{2}$, Nicky Cullum² \\ ${ }^{1}$ Departamento de Enfermería, Facultad de Enfermería de Melilla, Universidad de Granada, Granada, Spain. ${ }^{2}$ Division of Nursing, \\ Midwifery and Social Work, School of Health Sciences, Faculty of Biology, Medicine and Health, University of Manchester, Manchester \\ Academic Health Science Centre, Manchester, UK
}

Contact address: Rebeca Illescas-Montes, Departamento de Enfermería, Facultad de Enfermería de Melilla, Universidad de Granada, Granada, Spain. rebecaim@ugr.es, rebeca.illescasmontes@postgrad.manchester.ac.uk.

Editorial group: Cochrane Wounds Group.

Publication status and date: New, published in Issue 6, 2018.

Citation: Illescas-Montes R, Atkinson RA, Cullum N. Low-level light therapy for treating venous leg ulcers. Cochrane Database of Systematic Reviews 2018, Issue 6. Art. No.: CD013061. DOI: 10.1002/14651858.CD013061.

Copyright (C) 2018 The Cochrane Collaboration. Published by John Wiley \& Sons, Ltd.

\begin{abstract}
A B S T R A C T
This is a protocol for a Cochrane Review (Intervention). The objectives are as follows:

To determine the effects of low-level light therapy (LLLT) on the healing of venous leg ulcers.
\end{abstract}

\section{B A C K G ROU N D}

\section{Description of the condition}

Venous leg ulcers are chronic, open wounds usually located in the gaiter area of the lower leg (from below the ankle up to midcalf). These ulcers are caused by insufficiency of the deep or superficial veins, or both. The origin of venous insufficiency can be failure of valves or venous obstruction, caused by deep venous thrombosis for example. Venous insufficiency causes ambulatory venous hypertension, which leads to inflammation of the skin and ultimately skin breakdown. The exact chain of events from venous hypertension to venous ulceration is not fully clear, however several hypotheses have been developed (Coleridge 1988; Falanga 1993). People with venous leg ulcers frequently also have other comorbidities including arterial insufficiency, rheumatoid arthritis and diabetes (Moffatt 2004). Signs of venous insufficiency include varicose veins, dermal weeping, stasis dermatitis (swollen, red, itchy and painful skin), hyperpigmentation (skin darkening) and subcutaneous fibrosis (development of excess fibrous tissue under the skin) (Vasudevan 2014). Venous ulcers commonly have an irregular shape and well-defined borders; they can range in severity from partial- to full-thickness tissue loss (Nelzén 1994; Vasudevan 2014).

Global estimates of the point prevalence of leg ulceration range from $0.12 \%$ to $1.8 \%$; they are higher in women than men, and increase with age (Graham 2003). Recently, a cross-sectional study of the population of Leeds estimated the point prevalence of complex wounds to be 1.47 per 1000 population. In that survey, venous leg ulcers were the second most common complex wound with a point prevalence of 0.29 per 1000 population (Cullum 2016). The incidence of venous ulceration in the elderly population has been estimated at $0.76 / 100$ person-years for men, and 1.42/100 person-years for women (Margolis 2002).

The economic burden of complex wounds for the National Health Service in the UK has not been accurately estimated. In the UK, more than 9 million wound dressings were prescribed in the community in 2012, at a cost of 148 million GBP. However, the true cost of wound care is much greater due to the staff time and the cost of hospitalisation (the costs of which are much larger than 
the cost of dressings) (Cullum 2016). The cost of managing one person with a venous leg ulcer in the UK has been estimated to be approximately 1700 GBP per year at 2012 prices, where the highest cost component was nursing time (Ashby 2014).

Venous leg ulceration is a chronic condition, characterised by cycles of healing and recurrence with healing times ranging from weeks to years (Callam 1987). The longer the healing time, the greater the likelihood of decreased mobility, ankylosis (stiffening) of the ankle, wound infection and negative impact on quality of life (Parker 2015). Venous leg ulcers can also be malodorous and painful (Jones 2008), and are associated with sleep disorders and emotional distress (Green 2014).

The first-line treatment for venous leg ulcers is compression therapy, in the form of bandages or stockings (O'Meara 2012). Compression coupled with basic wound dressing use has been shown to heal approximately $68 \%$ of venous ulcers within 24 weeks (Iglesias 2004). More recently, new treatment options have been developed with the aim of improving these healing rates. New treatments include advanced wound dressings (O'Meara 2015; Sopata 2016), venous surgery (Van Gent 2015), bioengineered tissue (Jones 2013), laser therapy (Zhou 2016), and ultrasound (Cullum 2017).

\section{Description of the intervention}

Low-level light therapy (LLLT), also known as photobiomodulation (PBM), consists of the administration of light in specific conditions (low power conditions) with the purpose of promoting tissue repair. For decades, LLLT was regarded as solely associated with the use of lasers, however other light sources, such as Light Emitting Diodes (LEDs), are now also regarded as photobiomodulators and are another type of LLLT (Chung 2012; Chaves 2014). Any device that operates at an output power of less than 1 watt (W), or delivers treatment energies of less than $10 \mathrm{~J} / \mathrm{cm}^{2}$ (Basford 1989; Oltra-Arimon 2004), can be considered to be LLLT.

LLLT can be applied in a variety of ways. In general, the technique usually involves use of a handheld device, which administers a beam of light to a wound surface, without touching it. The treatment protocol varies according to duration of light application and the dose administered. The device can be moved over the wound in order to treat the whole surface (Mathur 2017). Standard wound care can be undertaken before and after each session. 'Laser' or 'Light Amplification by Stimulated Emission of Radiation' consists of light transmitted via an active medium. Maiman made the first functional laser in 1960; it had a ruby medium and a wavelength of $694.3 \mathrm{~nm}$ (Maiman 1960). Since then, lasers have been used widely across the field of medicine, initially in dermatology and ophthalmology (Götte 2001) with various types of laser available for use (De Freitas 2016). The active medium amplifies the light and can comprise a solid (e.g. neodymium-doped yttrium aluminium garnet laser - YAG, ruby laser), liquid or dye (e.g. coumarin, stilbene, rhodamine), gas (e.g. $\mathrm{CO}_{2}$, argon laser, helium-neon laser), or semiconductor (e.g. diode laser as gallium arsenide (GaAs), aluminium gallium arsenide (GaAlAs), indium gallium arsenide (InGaAs), or indium gallium arsenide phosphide (InGaAsP)). The active medium is placed between two mirrors: one that reflects completely and another that reflects partially. Electromagnetic waves move between these two mirrors, which act to amplify the energy (Kannatey-Asibu 2009).

The fundamentals of laser operation are based on the physical processes of stimulated emission, as described by Albert Einstein in 1916 (Einstein 1916). Stimulated emission is the process by which laser energy is produced (Kannatey-Asibu 2009). This energy can have many different characteristics, described in terms of power (usually in watts), energy (usually expressed as watts per second, or joules) and power density or fluence (measured in $\mathrm{J} / \mathrm{cm}^{2}$ ). Calculation of an exact dose of energy requires an understanding of the duration of time over which the energy will be administered. For example, to achieve a power density of $1 \mathrm{~J} / \mathrm{cm}^{2}$ and a mean output power of $0.2 \mathrm{~W}$, the light is applied for 7.7 seconds in a continuous wave mode, according to the formula: time $(\mathrm{s})=(\mathrm{J} /$ $\mathrm{cm}^{2} \mathrm{x}$ spot area)/W. Energy can be applied continuously, in an alternating mode, or a pulse mode. In continuous mode, an uninterrupted flow of energy at a specified energy level occurs while the laser is active. The alternating mode consists of alternate activation and deactivation of energy emission (using a shutter). Pulse mode occurs when energy is emitted at high power for a very short period followed by a much longer period of deactivation (Rossmann 1995; Karu 1997; Aoki 2004).

'NASA LED' (National Aeronautics and Space Administration LED) was the first type of LED phototherapy proposed as an alternative to laser therapy (Whelan 2000). In contrast to lasers, LEDs do not produce amplification of the light energy. Instead, they consist of a lamp light as a source of energy in the form of a photon. LEDs can emit visible red and near-infrared wavelengths. Energy is absorbed by the tissue receptor and produces a photochemical effect.

Healthcare applications of LLLT are generally classified on the basis of their potency and ability to interact with tissues. LLLT has characteristics such as biostimulation and can elicit a photochemical effect (i.e. the tissue absorbs light energy in order to produce a physical or chemical change, or both), making light energy a potentially suitable wound therapy (Conlan 1996).

\section{How the intervention might work}

The potential therapeutic effect of LLLT is based on the principle that light energy can lead to cell activation. Light (photon) energy is absorbed by cell molecule photoreceptors called cytochrome Coxidase (Cox), also called complex IV, of the mitochondrial respiratory chain. The enzymic change initiates biochemical pathways which, ultimately, increase cell activity (Karu 2008; Karu 2010). Other suggested mechanisms of action for LLLT in mediating cellular activity are via the impact of localised transient heating and 
nitric oxide modulation (Karu 2005; Karu 2008; Lane 2006). It is suggested that increased cellular activity along with associated cell proliferation could help in the tissue repair and regeneration process (Lins 2010; De Freitas 2016). Recent research in bone and connective tissue (Huertas 2014; Illescas-Montes 2017), and human skin cell Cox activity (Belletti 2015), has further elucidated the mechanism of action of LLLT.

\section{Why it is important to do this review}

Venous leg ulcers represent a serious health problem due to the complexity of the wound, the impact on people's quality of life and the high cost of treatment for health services. Current best treatments, such as compression, do not heal all venous ulcers and those that do heal can take weeks or months to heal completely or may not heal. The PBM effect of LLLT may increase the healing rates of venous leg ulcers. We have been unable to identify a highquality, up-to-date systematic review of the effects of LLLT on venous leg ulcer healing. We therefore concluded that a rigorous systematic review is required.

\section{O B JEC T IVES}

To determine the effects of low-level light therapy (LLLT) on the healing of venous leg ulcers.

\section{METHODS}

\section{Criteria for considering studies for this review}

\section{Types of studies}

We will include published and unpublished randomised controlled trials (RCTs), including cluster-RCTs, irrespective of the language of the report. We will only include cross-over trials if they report outcome data at the end of the first treatment period and before cross-over. Studies using quasi-randomisation will be excluded.

\section{Types of participants}

We will include RCTs recruiting people of any age with one or more venous leg ulcers, managed in any care setting. There will be no restrictions on trials due to participant age or the infection status of participants' wounds at baseline. We anticipate that the method of diagnosing venous leg ulceration may vary, so we will accept the definitions used by study authors.

Where studies enrol participants with other types of wounds (e.g. arterial leg ulcers, pressure ulcers, foot ulcers) as well as those with venous leg ulcers, we will include the study if the trial had a randomisation process that stratified based on wound type and the results for people with venous leg ulcers are presented separately. Where this is not the case we will only include the study if fewer than $25 \%$ of the participants have wounds other than venous leg ulcers.

\section{Types of interventions}

The primary intervention of interest is low-level light therapy (LLLT), including any device that operates at an output power less than 1 watt or power density less than $10 \mathrm{~J} / \mathrm{cm}^{2}$. We will include any RCT in which LLLT during the treatment period is the only systematic difference between intervention groups.

Eligible comparisons are as follows:

- LLLT versus no LLLT (in the form of usual care);

- LLLT versus sham LLLT;

- Different types of LLLT compared with each other;

- LLLT versus other alternative treatments.

\section{Types of outcome measures}

We list primary and secondary outcomes below. If a study is otherwise eligible (i.e. correct study design, population and intervention/comparator) but does not report a listed outcome, then we will contact the study authors where possible to establish whether an outcome of interest here was measured but not reported.

We will report outcomes at the latest time point available (assumed to be length of follow-up if not specified) and the time point specified in the methods as being of primary interest (if this is different from the latest time point available).

It is relevant to take time into account in the reporting of outcome measures. For all outcomes (primary and secondary) we will categorise assessment of outcome measures as:

- short term: up to and including eight weeks;

- medium term: more than eight weeks to up to and including 26 weeks;

- long term: more than 26 weeks.

\section{Primary outcomes}

The primary outcomes for this review are complete wound healing and adverse events.

- Complete wound healing

For this review, we will regard the following as providing the most relevant and rigorous measures of this outcome:

$\circ$ time to complete ulcer healing. We will record if this has been correctly analysed using censored data and with adjustment for prognostic covariates such as baseline size; $\circ$ the proportion of people with ulcers completely healed (frequency of complete healing). 
Where both of the outcomes above are reported we will present all data in a summary outcome table for reference, but will focus on reporting time to healing where it has been correctly analysed. We will accept authors' definitions of what constituted a healed wound.

- Adverse events

We will report events defined and grouped together as 'adverse events' by studies, where a clear methodology for the collection of adverse event data is provided. This methodology should make it clear whether: 1) events were reported at the participant level; or 2) if multiple events per person were reported. We anticipate that adverse events for LLLT will include wound infection, tissue deterioration, maceration (softening and breakdown) and pruritus (itching), which are similar to those for conventional treatments.

\section{Secondary outcomes}

Secondary outcomes are participant health-related quality of life, change in ulcer size, pain, wound infection and cost.

- Participant health-related quality of life

We will extract and analyse data on health-related quality of life where it has been measured using a standardised scale, such as the SF-36, SF-12, SF-6 or EQ-5D, or a validated disease-specific questionnaire such as the Cardiff Wound Impact Schedule (Guarnera 2007; Launois 2015). We will not include measures of quality of life that are unlikely to be validated and would not be common to multiple trials.

- Change in ulcer size

If there are no ulcer healing data for a particular comparison, we will consider using data on the change (and percentage change) in ulcer size, with adjustment for baseline size (we will contact study authors to request adjusted means when not presented). Where change in ulcer size is reported without adjustment for baseline size, use of the outcome in the study will be documented, but data will not be extracted, summarised or used in any meta-analysis.

- Pain

We will include pain scores only where they are reported either as presence or absence of pain during the care process, or as a continuous outcome using a validated scale such as a visual analogue scale (VAS) (Guarnera 2007).

- Wound infection

We will note whether wounds were infected at baseline and report, where possible, a resolution (or not) of the infection (efficacy) or incidence of infection (adverse events), or both.

- Cost

Mean costs associated with resource use and estimates of costeffectiveness.

\section{Search methods for identification of studies}

\section{Electronic searches}

We will search the following electronic databases to retrieve reports of relevant clinical trials:

- Cochrane Wounds Group Specialised Register;

- Cochrane Central Register of Controlled Trials

(CENTRAL) (the Cochrane Library, latest issue);

- Ovid MEDLINE (from 1946 onwards);

- Ovid MEDLINE (In-Process \& Other Non-Indexed

Citations);

- Ovid Embase (from 1974 onwards);

- EBSCO CINAHL Plus (Cumulative Index to Nursing and Allied Health Literature; from 1937 onwards).

We have devised a draft search strategy for CENTRAL which is presented in Appendix 1. We will adapt this strategy to search Ovid MEDLINE, Ovid Embase and EBSCO CINAHL Plus. We will combine the Ovid MEDLINE search with the Cochrane Highly Sensitive Search Strategy for identifying randomised trials in MEDLINE: sensitivity- and precision-maximising version (2008 revision) (Lefebvre 2011). We will combine the Embase search with the Ovid Embase filter developed by the UK Cochrane Centre (Lefebvre 2011). We will combine the CINAHL Plus search with the trial filters developed by the Scottish Intercollegiate Guidelines Network (SIGN 2018). There will be no restrictions with respect to language, date of publication or study setting. We will also search the following clinical trials registries:

- ClinicalTrials.gov (www.clinicaltrials.gov);

- World Health Organization (WHO) International Clinical Trials Registry Platform (www.who.int/trialsearch);

- EU Clinical Trials Register (www.clinicaltrialsregister.eu/ ctr-search/search).

\section{Searching other resources}

We aim to identify other potentially eligible trials or ancillary publications by searching the reference lists of retrieved included trials as well as relevant systematic reviews, meta-analyses, and health-technology assessment reports.

\section{Data collection and analysis}

\section{Selection of studies}

Two review authors will independently assess the titles and abstracts of the citations retrieved by the searches for relevance. After this initial assessment, we will obtain full-text copies of all studies considered to be potentially relevant. Two review authors will independently check the full papers for eligibility; disagreements 
will be resolved by discussion and, where required, the input of a third review author. Where required and possible, we will contact study authors where the eligibility of a study is unclear. We will record all reasons for exclusion of studies for which we had obtained full copies. We will complete a PRISMA (Preferred Reporting Items for Systematic Reviews and Meta-Analyses) flowchart to summarise this process (Liberati 2009).

Where studies have been reported in multiple publications/reports, we will obtain all publications. Whilst we will include the study only once in the review, we will extract data from all reports to ensure maximal relevant data are obtained.

\section{Data extraction and management}

We will extract and summarise details of eligible studies using a data extraction sheet. Two review authors will extract data independently and will resolve disagreements by discussion, consulting a third review author where required. Where data are missing from reports, we will attempt to contact the study authors to obtain this information. Where a study with more than two intervention arms is included, we will only extract data from intervention and control groups that meet the eligibility criteria.

We will extract the following data:

- country in which the study was conducted;

- unit of randomisation: cluster, participant, wounds (for split-site or split-body study), leg;

- trial design, e.g. parallel, cluster, ulcer randomisation, crossover trials with first period results;

- publication status of study;

- source of funding;

- care setting;

- number of participants randomised to each trial arm;

- inclusion and exclusion criteria;

- population baseline characteristics:

o age

o sex

- duration of venous leg ulcer

- ulcer area at baseline

- proportion of patients with infected ulcers at baseline;

- treatment received by each group:

$\circ$ details of treatment regimen (e.g. wavelength and type of device)

$\circ$ protocol of treatment delivery (e.g. dose, frequency)

o details of any co-interventions

o duration of treatment

duration of follow-up

- unit of analysis and details of analysis;

- outcome data for primary and secondary outcomes (by group);

- number of withdrawals per group and reasons for withdrawal.

\section{Assessment of risk of bias in included studies}

Two review authors will independently assess included studies using the Cochrane tool for assessing risk of bias (Higgins 2011a). This tool addresses seven specific domains: random sequence generation (selection bias), allocation concealment (selection bias), blinding of participants and personnel (performance bias), blinding of outcome assessment (detection bias), incomplete outcome data (attrition bias), selective outcome reporting bias and other issues leading to bias (e.g. unit of analysis issues).

We will assess blinding and completeness of outcome data for each of the review outcomes separately. We note that, since wound healing is a subjective outcome, it can be at high risk of detection bias when outcome assessment is not blinded. For blinding of participants and personnel (performance bias), use of sham LLLT is likely to lead to a low risk of bias assessment if it blinds those giving and receiving treatment. For open studies, where participants and personnel will have knowledge of treatment received, any potential differences in care pathways between groups that might lead to bias will be considered. We will present our assessment of risk of bias using two 'Risk of bias' summary figures; one which is a summary of bias for each item across all studies, and the second of which shows a cross-tabulation of each trial by all of the 'Risk of bias' items. We will class studies as being at overall high risk of bias if they are rated at 'high risk' for any of the domains.

We will class studies at low risk of bias if they are judged to be at low risk of selection, detection and attrition bias (with no domains at high risk of bias).

\section{Measures of treatment effect}

Time-to-event data (e.g. time to complete wound healing) will be reported as hazard ratios (HR) where possible in accordance with the methods described in the Cochrane Handbook for Systematic Reviews of Interventions (Deeks 2011). If studies reporting timeto-event data (e.g. time to healing) do not report a hazard ratio, then, where feasible, we plan to estimate this using other reported outcomes, such as the numbers of events, through the application of available statistical methods (Parmar 1998). We will only consider mean or median time to healing without survival analysis as a valid outcome if reports specify that all wounds healed (i.e. if the trial authors regarded time to healing as a continuous measure as there is no censoring).

For dichotomous outcomes we will calculate the risk ratio (RR) with $95 \%$ confidence intervals (CIs). For continuous outcome data we will use the mean difference (MD) with $95 \%$ CIs.

\section{Unit of analysis issues}

Where studies randomise at the participant level and measure outcomes at the wound level, e.g. wound healing, we will treat the participant as the unit of analysis when the number of wounds assessed appears equal to the number of participants (e.g. one wound per person). 
Particular unit of analysis issues in wound care trials can occur when studies randomise at the participant level and then use the allocated treatment on multiple wounds per participant, and then analyse outcomes per wound, not person. This is a type of clustered data and presents a unit of analysis error that inflates precision. In cases where included studies contained some or only clustered data, we plan to report this alongside whether data had been (incorrectly) treated as independent. We will record this as part of the 'Risk of bias' assessment. We do not plan to undertake further calculation to adjust for clustering where it has not been considered in the study.

\section{Dealing with missing data}

It is common for there to be data missing from trial reports. Excluding participants from the analysis after randomisation, or ignoring those participants who are lost to follow-up, compromises the randomisation and potentially introduces bias into the trial. Where there are missing data we think should be included in the analyses, we will contact the relevant study authors to request whether these data are available.

Where data remain missing for the 'proportion of wounds healed' outcome, for analysis we will assume that if randomised participants were not included in an analysis, their wound did not heal (i.e. they would be considered in the denominator but not the numerator).

In a time-to-healing analysis using survival analysis methods, dropouts should be accounted for as censored data, so no action regarding missing data will be taken.

For continuous variables, e.g. length of hospital stay, and for all secondary outcomes, we will present available data from the study reports/study authors and do not plan to impute missing data. Where measures of variance are missing we will calculate these wherever possible (Higgins 2011b). If calculation is not possible we will contact the study authors. Where these measures of variance are not available, we will exclude the study from any relevant metaanalyses that are conducted.

\section{Assessment of heterogeneity}

Assessment of heterogeneity can be a complex, multi-faceted process. Firstly, we will consider clinical and methodological heterogeneity: that is the degree to which the included studies vary in terms of participant, intervention, outcome and characteristics such as length of follow-up. This assessment of clinical and methodological heterogeneity will be supplemented by information regarding statistical heterogeneity, assessed using the $\mathrm{Chi}^{2}$ test (a significance level of $\mathrm{P}<0.10$ will be considered to indicate statistically significant heterogeneity) in conjunction with the $\mathrm{I}^{2}$ measure (Higgins 2003). $\mathrm{I}^{2}$ examines the percentage of total variation across RCTs that is due to heterogeneity rather than chance (Higgins 2003). In general, $\mathrm{I}^{2}$ values of $25 \%$ or less may mean a low level of heterogeneity (Higgins 2003), and values of more than $75 \%$ indicate very high heterogeneity (Deeks 2011). Where there is evidence of high heterogeneity we will attempt to explore this further: see Data synthesis.

\section{Assessment of reporting biases}

Reporting biases arise when the dissemination of research findings is influenced by the nature and direction of results. Publication bias is one of a number of possible causes of 'small study effects', that is, a tendency for estimates of the intervention effect to be more beneficial in smaller RCTs. Funnel plots allow a visual assessment of whether small study effects may be present in a meta-analysis. A funnel plot is a simple scatter plot of the intervention effect estimates from individual RCTs against some measure of each trial's size or precision (Sterne 2011). We plan to present funnel plots for meta-analyses comprising 10 or more RCTs using Review Manager 5 (Review Manager 2014).

\section{Data synthesis}

We will combine details of included studies in a narrative review according to type of comparator, possibly by intervention, and then by outcomes by time point.

Clinical and methodological heterogeneity will be considered and pooling undertaken when studies appear appropriately similar in terms of intervention type, duration of follow-up and outcome type.

We are unable to pre specify the amount of clinical, methodological and statistical heterogeneity in the included studies, but it might be extensive. Thus, we anticipate using a random-effects approach for meta-analysis. Conducting meta-analysis with a fixedeffect model in the presence of even minor heterogeneity may provide overly narrow confidence intervals. We will only use a fixedeffect approach when clinical and methodological heterogeneity is assessed to be minimal, and the assumption that a single underlying treatment effect is being estimated holds. We will use Chi 2 and $\mathrm{I}^{2}$ to quantify heterogeneity but this will not guide choice of model for meta-analysis. We will exercise caution when metaanalysed data are at risk of small study effects because a randomeffects model may be unsuitable. In this case, or where there are other reasons to question the selection of a fixed-effect or randomeffects model, we will assess the impact of the approach using sensitivity analyses to compare results from alternate models. We will report any evidence that suggests that use of a particular model might not be robust. We may meta-analyse even when there is thought to be extensive heterogeneity (Deeks 2011). We will attempt to explore the causes behind this using meta-regression for that purpose, if possible (Thompson 1999).

We will present data using forest plots where possible. For dichotomous outcomes we will present the summary estimate as a risk ratio (RR) with $95 \%$ CI. Where continuous outcomes are measured 
in the same way across studies, we plan to present a pooled mean difference (MD) with 95\% CI. If trials use different assessment scales, we will use the standardised mean difference (SMD) with 95\% CIs. For interpretation, SMDs will be converted to the MD using an appropriate scale. For time-to-event data, we plan to plot (and, if appropriate, pool) estimates of hazard ratios and 95\% CIs as presented in the study reports using the generic inverse variance method in Review Manager 5. Where time to healing is analysed as a continuous measure but it is not clear if all wounds healed, use of the outcome in the study will be documented but data will not be summarised or used in any meta-analysis.

Pooled estimates of treatment effect will be obtained using Review Manager 5 software (Review Manager 2014).

\section{'Summary of findings' tables and assessment of the quality of the evidence using the GRADE approach}

We will present the main results of the review in 'Summary of findings' tables. These tables present key information concerning the quality of the evidence, the magnitude of the effects of the interventions examined, and the sum of the available data for the main outcomes (Schünemann 2011a). The 'Summary of findings' tables also include an overall grading of the evidence related to each of the main outcomes using the GRADE (Grades of Recommendation, Assessment, Development and Evaluation) approach. The GRADE approach defines the quality of a body of evidence as the extent to which one can be confident that an estimate of effect or association is close to the true quantity of specific interest. The quality of a body of evidence involves consideration of withintrial risk of bias (methodological quality), directness of evidence, heterogeneity, precision of effect estimates and risk of publication bias (Schünemann 2011b). We will present the following primary outcomes in the 'Summary of findings' tables:

- time to complete healing;

- proportion of peopled with ulcers completely healed;

- adverse events.
For relevant outcomes reported for comparisons not listed above we will present GRADE assessments narratively within the Results section, without a 'Summary of findings' table.

In terms of the GRADE assessment, when making decisions for the 'Risk of bias' domain, we will consider downgrading only when studies are classed as having high risk of bias for one or more domains. We will not downgrade for unclear risk of bias assessments. In assessing the precision of effect estimates, we will also follow GRADE guidance (GRADE 2013); we will assess the size of confidence intervals, downgrading twice for imprecision when there are very few events and CIs around effects include both appreciable benefit and appreciable harm.

\section{Subgroup analysis and investigation of heterogeneity}

We plan to perform subgroup analyses to explore the effect depending on the type of light source, except in cases where the subgroup consists of a single study. We will explore differences in the response of three possible types of intervention: laser therapy; LED phototherapy; and laser therapy combined with LED phototherapy.

\section{Sensitivity analysis}

Where possible we will repeat meta-analyses with studies removed if they are considered to be at high risk of bias, with the aim of trying to explain and explore heterogeneity.

Elements of this Methods section are based on the standard Cochrane Wounds protocol template.

\section{ACKNOWLEDGEMENTS}

The authors would like to thank peer reviewers Susan O'Meara (Editor), Nicolette Houreld, Antonia Marsden and Janet Gunderson for their comments on the protocol. Thanks are also due to Jessica Sharp for copy-editing the protocol.

\section{REFEREN CES}

\section{Additional references}

\section{Aoki 2004}

Aoki A, Sasaki KM, Watanabe H, Ishikawa I. Lasers in nonsurgical periodontal therapy. Periodontology 2000 2004; 36:59-97.

Ashby 2014

Ashby RL, Gabe R, Ali S, Adderley U, Bland JM, Cullum NA, et al. Clinical and cost-effectiveness of compression hosiery versus compression bandages in treatment of venous leg ulcers (Venous leg Ulcer Study IV, VenUS IV): a randomised controlled trial. Lancet 2014;383(9920):

871-9.

Basford 1989

Basford JR. Low-energy laser therapy: controversies and new research findings. Lasers in Surgery and Medicine 1989; 9(1):1-5.

\section{Belletti 2015}

Belletti S, Uggeri J, Mergoni G, Vescovi P, Merigo E, Fornaini $\mathrm{C}$, et al. Effects of $915 \mathrm{~nm}$ GaAs diode laser on mitochondria of human dermal fibroblasts: analysis with 
confocal microscopy. Lasers in Medical Science 2015;30(1): 375-81.

\section{Callam 1987}

Callam MJ, Harper DR, Dale JJ, Ruckley CV. Chronic ulcer of the leg: clinical history. British Medical Journal 1987;294(6584):1389-91.

Chaves 2014

Chaves ME, Araújo AR, Piancastelli AC, Pinotti M. Effects of low-power light therapy on wound healing: LASER $x$ LED. Anais Brasileiros de Dermatologia 2014;89(4):616-23.

\section{Chung 2012}

Chung H, Dai T, Sharma S, Huang Y, Carroll J, Hamblin $\mathrm{M}$. The nuts and bolts of low-level laser (light) therapy. Annals of Biomedical Engineering 2012;40(2):516-33.

\section{Coleridge 1988}

Coleridge Smith PD, Thomas P, Scurr JH, Dormandy JA. Causes of venous ulceration: a new hypothesis. British Medical Journal 1988;296(6638):1726-7.

\section{Conlan 1996}

Conlan MJ, Rapley JW, Cobb CM. Biostimulation of wound healing by low-energy laser irradiation. A review. Journal of Clinical Periodontology 1996;23(5):492-6.

\section{Cullum 2016}

Cullum N, Buckley H, Dumville J, Hall J, Lamb K, Madden M, et al. Wounds research for patient benefit: a 5year programme of research. Programme Grants for Applied Research 2016;4(13):1-334.

\section{Cullum 2017}

Cullum N, Liu Z. Therapeutic ultrasound for venous leg ulcers. Cochrane Database of Systematic Reviews 2017, Issue 5. DOI: 10.1002/14651858.CD001180.pub4

De Freitas 2016

De Freitas LF, Hamblin MR. Proposed mechanisms of photobiomodulation or low-level light therapy. IEEE Journal of Selected Topics in Quantum Electronics 2016;22 (3):7000417.

Deeks 2011

Deeks JJ, Higgins JP, Altman DG editor(s), on behalf of the Cochrane Statistical Methods Group. Chapter 9: Analysing data and undertaking meta-analyses. In: Higgins JP, Green S, editor(s). Cochrane Handbook for Systematic Reviews of Interventions Version 5.1.0 (updated March 2011). The Cochrane Collaboration, 2011. Available from handbook.cochrane.org.

\section{Einstein 1916}

Einstein A. Emission and absorption of radiation in quantum theory [Strahlungs-Emission und Absorption nach der Quantentheorie]. Deutsche Physikalische Gesellschaft 1916;18:318-23.

\section{Falanga 1993}

Falanga V, Eaglstein WH. The "trap" hypothesis of venous ulceration. Lancet 1993;341(8851):1006-8.

\section{GRADE 2013}

Schünemann H, Broż ek J, Guyatt G, Oxman A, GRADE working group. GRADE handbook. October 2013. gdt.guidelinedevelopment.org/central_prod/_design/client/ handbook/handbook.html (accessed 7 December 2017).

\section{Graham 2003}

Graham ID, Harrison MB, Nelson EA, Lorimer K, Fisher A. Prevalence of lower-limb ulceration: a systematic review of prevalence studies. Advances in Skin \& Wound Care 2003; 16(6):305-16.

\section{Green 2014}

Green J, Jester R, McKinley R, Pooler A. The impact of chronic venous leg ulcers: a systematic review. Journal of Wound Care 2014;23(12):601-12.

\section{Guarnera 2007}

Guarnera G, Tinelli G, Abeni D, Di Pietro C, Sampogna F, Tabolli S. Pain and quality of life in patients with vascular leg ulcers: an Italian multicentre study. Journal of Wound Care 2007;16(8):347-51.

Götte 2001

Götte S. Low-level laser therapy. Lasers in the Musculoskeletal System. Berlin: Springer, 2001:199-204.

\section{Higgins 2003}

Higgins JP, Thompson SG, Deeks JJ, Altman DG. Measuring inconsistency in meta-analyses. BMJ 2003;327 (7414):557-60.

\section{Higgins 2011a}

Higgins JP, Altman DG, Sterne JA, editor(s). Chapter 8: Assessing risk of bias in included studies. In: Higgins JP, Green S, editor(s). Cochrane Handbook for Systematic Reviews of Interventions Version 5.1.0 (updated March 2011). The Cochrane Collaboration, 2011. Available from handbook.cochrane.org.

\section{Higgins 2011b}

Higgins JP, Deeks JJ, editor(s). Chapter 7: Selecting studies and collecting data. In: Higgins JP, Green S, editor(s). Cochrane Handbook for Systematic Reviews of Interventions Version 5.1.0 (updated March 2011). The Cochrane Collaboration, 2011. Available from handbook.cochrane.org.

\section{Huertas 2014}

Huertas RM, Luna-Bertos ED, Ramos-Torrecillas J, Leyva FM, Ruiz C, García-Martínez O. Effect and clinical implications of the low-energy diode laser on bone cell proliferation. Biological Research for Nursing 2014;16(2): 191-6.

\section{Iglesias 2004}

Iglesias C, Nelson EA, Cullum NA, Torgerson DJ, VenUS Team. VenUS I: a randomised controlled trial of two types of bandage for treating venous leg ulcers. Health Technology Assessment 2004;8(29):iii,-1-105.

\section{Illescas-Montes 2017}

Illescas-Montes R, Melguizo-Rodríguez L, ManzanoMoreno FJ, García-Martínez O, Ruiz C, Ramos-Torrecillas J. Cultured human fibroblast biostimulation using a 940 nm diode laser. Materials 2017;10(7):E793. 
Jones 2008

Jones JE, Robinson J, Barr W, Carlisle C. Impact of exudate and odour from chronic venous leg ulceration. Nursing Standard 2008;22(45):53-54, 56, 58 passim.

\section{Jones 2013}

Jones JE, Nelson EA, Al-Hity A. Skin grafting for venous leg ulcers. Cochrane Database of Systematic Reviews 2013, Issue 1. DOI: 10.1002/14651858.CD001737.pub4

\section{Kannatey-Asibu 2009}

Kannatey-Asibu E. Principles of Laser Materials Processing. Wiley, 2009.

\section{Karu 1997}

Karu TI, Ryabykh TP, Letokhov VS. Different sensitivity of cells from tumor- bearing organisms to continuouswave and pulsed laser radiation $(632,8 \mathrm{~nm})$ evaluated by chemiluminescence test. Photobiology 1997;7:141-56.

\section{Karu 2005}

Karu TI, Pyatibrat LV, Afanasyeva NI. Cellular effects of low power laser therapy can be mediated by nitric oxide. Lasers in Surgery and Medicine 2005;36(4):307-14.

\section{Karu 2008}

Karu TI. Mitochondrial signaling in mammalian cells activated by red and near-IR radiation. Photochemistry and Photobiology 2008;84(5):1091-9.

\section{Karu 2010}

Karu TI. Multiple roles of cytochrome c oxidase in mammalian cells under action of red and IR-A radiation. IUBMB Life 2010;62(8):607-10.

\section{Lane 2006}

Lane N. Cell biology: power games. Nature 2006;443 (7114):901-3.

\section{Launois 2015}

Launois R. Health-related quality-of-life scales specific for chronic venous disorders of the lower limbs. Journal of Vascular Surgery. Venous and Lymphatic Disorders 2015;3(2): 219-27.

\section{Lefebvre 2011}

Lefebvre C, Manheimer E, Glanville J. Chapter 6: Searching for studies. In: Higgins JP, Green S, editor(s). Cochrane Handbook for Systematic Reviews of Interventions Version 5.1.0 (updated March 2011). The Cochrane Collaboration, 2011. Available from handbook.cochrane.org.

\section{Liberati 2009}

Liberati A, Altman DG, Tetzlaff J, Mulrow C, Gøtzsche PC, Ioannidis JP, et al. The PRISMA statement for reporting systematic reviews and meta-analyses of studies that evaluate healthcare interventions: explanation and elaboration. $B M J$ 2009;339:b2700.

Lins 2010

Lins, Ruthinéia Diógenes Alves Uchôa, Dantas, Euler Maciel, Lucena, Keila Cristina Raposo, et al. Biostimulation effects of low-power laser in the repair process. Anais Brasileiros de Dermatologia 2010; Vol. 85, issue 6:849-55.

\section{Maiman 1960}

Maiman TH. Stimulated optical radiation in ruby. Nature 1960;187(4736):493-4.

\section{Margolis 2002}

Margolis DJ, Bilker W, Santanna J, Baumgarten M. Venous leg ulcer: incidence and prevalence in the elderly. Journal of the American Academy of Dermatology 2002;46(3):381-6.

\section{Mathur 2017}

Mathur RK, Sahu K, Saraf S, Patheja P, Khan F, Gupta PK. Low-level laser therapy as an adjunct to conventional therapy in the treatment of diabetic foot ulcers. Lasers in Medical Science 2017;32(2):275-82.

\section{Moffatt 2004}

Moffatt CJ, Franks PJ, Doherty DC, Martin R, Blewett R, Ross F. Prevalence of leg ulceration in a London population. QJM 2004;97(7):431-7.

\section{Nelzén 1994}

Nelzén O, Bergqvist D, Lindhagen A. Venous and nonvenous leg ulcers: clinical history and appearance in a population study. British Journal of Surgery 1994;81(2): $182-7$.

\section{O'Meara 2012}

O'Meara S, Cullum N, Nelson EA, Dumville JC. Compression for venous leg ulcers. Cochrane Database of Systematic Reviews 2012, Issue 11. DOI: 10.1002/ 14651858.CD000265.pub3

\section{O'Meara 2015}

O'Meara S, Martyn-St JM, Adderley UJ. Alginate dressings for venous leg ulcers. Cochrane Database of Systematic Reviews 2015, Issue 8. DOI: 10.1002/ 14651858.CD010182.pub3

\section{Oltra-Arimon 2004}

Oltra-Arimon D, España-Tost AJ, Berini-Aytés L, GayEscoda C. Applications of low level laser therapy in dentistry [Aplicaciones del láser de baja potencia en odontología]. RCOE [Revista del Ilustre Consejo General de Colegios de Odontólogos y Estomatólogos de España] 2004;9(5):517-24.

Parker 2015

Parker CN, Finlayson KJ, Shuter P, Edwards HE. Risk factors for delayed healing in venous leg ulcers: a review of the literature. International Journal of Clinical Practice 2015; 69(9):967-77.

\section{Parmar 1998}

Parmar MK, Torri V, Stewart L. Extracting summary statistics to perform meta-analyses of the published literature for survival endpoints. Statistics in Medicine 1998;17(24): 2815-34.

Review Manager 2014 [Computer program] Nordic Cochrane Centre, The Cochrane Collaboration. Review Manager 5 (RevMan 5) 5.3. Copenhagen: Nordic Cochrane Centre, The Cochrane Collaboration, 2014.

\section{Rossmann 1995}

Rossmann JA, Cobb CM. Lasers in periodontal therapy. Periodontology 2000 1995;9:150-64. 
Schünemann 2011a

Schünemann HJ, Oxman AD, Higgins JP, Vist GE, Glasziou P, Akl E, et al. Chapter 11: Completing 'Summary of findings' tables and grading the confidence in or quality of the evidence. In: Higgins JP, Green S, editor(s). Cochrane Handbook for Systematic Reviews of Interventions Version 5.1.0 (updated March 2011). The Cochrane Collaboration, 2011. Available from handbook.cochrane.org.

\section{Schünemann 2011b}

Schünemann HJ, Oxman AD, Vist GE, Higgins JP, Deeks JJ, Glasziou P, et al. Chapter 12: Interpreting results and drawing conclusions. In: Higgins JP, Green S, editor(s). Cochrane Handbook for Systematic Reviews of Interventions Version 5.1.0 (updated March 2011). The Cochrane Collaboration, 2011. Available from handbook.cochrane.org.

\section{SIGN 2018}

Scottish Intercollegiate Guidelines Network (SIGN). Search filters. www.sign.ac.uk/search-filters.html (accessed 8 June 2018).

\section{Sopata 2016}

Sopata M, Kucharzewski M, Tomaszewska E. Antiseptic with modern wound dressings in the treatment of venous leg ulcers: clinical and microbiological aspects. Journal of Wound Care 2016;25(8):419-26.

\section{Sterne 2011}

Sterne JA, Egger M, Moher D, Boutron I, editor(s). Chapter 10: Addressing reporting biases. In: Higgins JP, Green S, editor(s). Cochrane Handbook for Systematic
Reviews of Interventions Version 5.1.0 (updated March 2011). The Cochrane Collaboration, 2011. Available from handbook.cochrane.org.

\section{Thompson 1999}

Thompson SG, Sharp SJ. Explaining heterogeneity in metaanalysis: a comparison of methods. Statistics in Medicine 1999;18(20):2693-708.

Van Gent 2015

Van Gent WB, Catarinella FS, Lam YL, Nieman FH, Toonder IM, Van der Ham AC, et al. Conservative versus surgical treatment of venous leg ulcers: 10-year follow up of a randomized, multicenter trial. Phlebology 2015;30(Suppl 1):35-41.

\section{Vasudevan 2014}

Vasudevan B. Venous leg ulcers: pathophysiology and classification. Indian Dermatology Online Journal 2014;5 (3):366-70.

\section{Whelan 2000}

Whelan HT, Houle JM, Whelan NT, Donohoe DL, Cwiklinski J, Schmidt MH, et al. The NASA light-emitting diode medical program - progress in space flight and terrestrial applications. AIP Conference Proceedings 2000; 504(1):37-43.

\section{Zhou 2016}

Zhou Y-T, Zhao X-D, Jiang J-W, Li X-S, Wu Z-H. Ozone gas bath combined with endovenous laser therapy for lower limb venous ulcers: a randomized clinical trial. Journal of Investigative Surgery 2016;29(5):254-9.

* Indicates the major publication for the study

\section{AP PENDICES}

\section{Appendix I. The Cochrane Central Register of Controlled Trials (CENTRAL) draft search strategy}

\#1 MeSH descriptor: [Leg Ulcer] explode all trees

\#2 ((varicose next ulcer*) or (venous near/2 ulcer*) or (leg next ulcer*) or (stasis next ulcer*) or (crural next ulcer*) or "ulcus cruris" or "ulcer cruris" or ((lower next extremit*) near/2 ulcer*)):ti,ab,kw

\#3 \#1 or \#2

\#4 MeSH descriptor: [Laser Therapy] explode all trees

\#5 MeSH descriptor: [Low-Level Light Therapy] explode all trees

\#6 MeSH descriptor: [Phototherapy] explode all trees

\#7 MeSH descriptor: [Infrared Rays] explode all trees

\#8 MeSH descriptor: [Lasers] explode all trees

\#9 (LLLT):ti,ab,kw

\#10 (LEPT):ti,ab,kw

\#11 (LPLT):ti,ab,kw

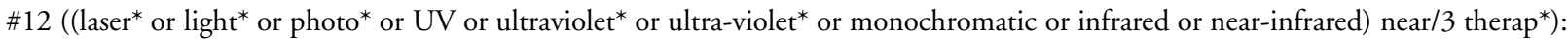
ti,ab,kw

Low-level light therapy for treating venous leg ulcers (Protocol)

Copyright ( 2018 The Cochrane Collaboration. Published by John Wiley \& Sons, Ltd. 


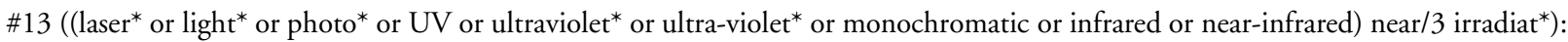
ti,ab,kw

\#14 ((laser* or light* or photo* or UV or ultraviolet* or ultra-violet* or monochromatic or infrared or near-infrared) near/3 biostimulat*): ti,ab,kw

\#15 ((laser* or light* or photo* or UV or ultraviolet* or ultra-violet* or monochromatic or infrared or near-infrared) near/3 stimulat*): ti,ab, kw

\#16 (low* near/3 laser*):ti,ab,kw

\#17 (phototherap* or photo-therap*):ti,ab,kw

\#18 (photobiomodulation or photo-biomodulation or photobiotherap* or photo-biotherap*):ti,ab,kw

\#19 (photobiostimulat* or photo-biostimulat*):ti,ab,kw

\#20 LED?:ti,ab,kw

\#21 (light-emitting diode* or light emitting diode*):ti,ab,kw

\#22 ((diode near/3 laser*) or (photodiode*) or (photo-diode*)):ti,ab,kw

\#23 ((helium next neon) or (helium-neon) or HeNe or He-Ne):ti,ab,kw

\#24 ((gallium next arsenide) or (gallium-arsenide) or GaAs or Ga-As):ti,ab,kw

\#25 ((gallium next alumin*) or (gallium-alumin*) or GaAIAs):ti,ab,kw

\#26 ((gallium near/2 indium) or (gallium-indium) or (indium-gallium) or InGaAs or InGaAsP):ti,ab,kw

\#27 ((neodynium next doped) or (neodynium-doped) or Nd-YAG or NdYAG or Nd YAG):ti,ab,kw

$\# 28 \# 4$ or \#5 or \#6 or \#7 or \#8 or \#9 or \#10 or \#11 or \#12 or \#13 or \#14 or \#15 or \#16 or \#17 or \#18 or \#19 or \#20 or \#21 or \#22 or

$\# 23$ or \#24 or \#25 or \#26 or \#27

$\# 29 \# 3$ and \#28

\section{CONTRIBUTIONSOFAUTHORS}

Rebeca Illescas-Montes: conceived the review question; developed the protocol; produced the first draft of the protocol; contributed to writing and editing the protocol; approved the final version of the protocol prior to submission; and is guarantor of the protocol.

Nicky Cullum: conceived the review question; co-ordinated the protocol development; secured funding; produced the first draft of the protocol; advised on the protocol; and approved the final version of the protocol prior to submission.

Ross Atkinson: developed the protocol; co-ordinated the protocol development; contributed to writing and editing the protocol; approved the final version of the protocol prior to submission.

\section{Contributions of Editorial base}

Jo Dumville (Co-ordinating Editor): edited the protocol; advised on methodology, interpretation and protocol content; approved the final version of the protocol prior to submission.

Gill Rizzello (Managing Editor): co-ordinated the editorial process; advised on content and edited the protocol.

Naomi Shaw (Information Specialist): designed the search strategy and edited the search methods section.

Ursula Gonthier (Editorial Assistant): edited the reference section.

\section{DECLARATIONSOF INTEREST}

Rebeca Illescas-Montes: none known.

Nicky Cullum: received research funding for wounds-related research and systematic reviews from the NIHR for the production of systematic reviews focusing on high-priority Cochrane Reviews in the prevention and treatment of wounds. This research was co-funded by the NIHR Manchester Biomedical Research Centre, and partly funded by the National Institute for Health Research Collaboration for Leadership in Applied Health Research and Care (NIHR CLAHRC) Greater Manchester.

Ross Atkinson: none known.

Low-level light therapy for treating venous leg ulcers (Protocol) 


\section{SOURCES OF SUPPORT}

\section{Internal sources}

- Division of Nursing, Midwifery and Social Work, School of Health Sciences, Faculty of Biology, Medicine and Health, University of Manchester, UK.

\section{External sources}

- National Institute for Health Research (NIHR), UK.

This project was supported by the NIHR, via Cochrane Infrastructure and Cochrane Programme Grant funding (NIHR Cochrane Programme Grant 13/89/08 - High Priority Cochrane Reviews in Wound Prevention and Treatment) to Cochrane Wounds. The views and opinions expressed herein are those of the authors and do not necessarily reflect those of the Systematic Reviews Programme, the NIHR, the NHS or the Department of Health.

- NIHR Manchester Biomedical Research Centre (BRC), UK.

This research was co-funded by the NIHR Manchester BRC. The views expressed in this publication are those of the authors and not necessarily those of the NHS, the National Institute for Health Research or the Department of Health.

- National Health Research Collaboration for Leadership in Applied Health Research and Care (NIHR CLAHRC), Greater Manchester, UK.

Nicky Cullum's work on this project was partly funded by the NIHR CLAHRC, Greater Manchester. The funder had no role in the decision to publish, or preparation of this manuscript. However, the review may be considered to be affiliated to the work of the NIHR CLAHRC Greater Manchester. The views expressed herein are those of the authors and not necessarily those of the NHS, NIHR or the Department of Health. 